\title{
Tailor-made molecularly imprinted polymers for dimethoate and deltamethrin recognition: synthesis, characterization and chromatographic evaluation
}

\author{
Marco Simões • Nuno Martins • Maria João Cabrita • \\ Anthony J. Burke $\cdot$ Raquel Garcia
}

Received: 23 August 2013 / Accepted: 22 January 2014

(C) Springer Science+Business Media Dordrecht 2014

\begin{abstract}
This work concerns the development of molecularly imprinted polymers (MIPs) for the selective extraction of dimethoate $(\mathrm{dmt})$ and deltamethrin $(\mathrm{dm})$ from food matrices. To achieve this goal, the non-covalent methodology has been applied for the preparation of MIPs using metacrylic acid (MAA) as a functional monomer and ethylene glycol dimethacrylate (EGDMA) and triethylene glycol dimethacrylate (TEGDMA) as cross-linkers in order to evaluate the influence of the nature of the cross-linker on the efficiency and selectivity of those MIPs for the target pesticides. Non-imprinted polymers (NIPs), which do not contain template, have been also prepared in parallel with the MIP synthesis using the same synthetic protocol to assess the specificity of the interactions. Chemical and physical characterization was carried out using conventional techniques, such as Fourier transform infrared (FTIR) spectroscopy, nuclear magnetic resonance (NMR) spectroscopy and thermogravimetric analysis (TGA). Morphological characterization of MIPs and NIPs has been also performed using scanning electron microscopy (SEM) in order to assess the polymer's surface topography. The performance of each polymer was evaluated by conducting binding property measurements, namely imprinting factor determinations and adsorption studies using high performance liquid chromatography (HPLC).
\end{abstract}

\footnotetext{
M. Simões · A. J. Burke

Departamento de Química e Centro de Química de Évora,

Universidade de Évora, Rua Romão Ramalho, 597000 Évora,

Portugal

N. Martins $\cdot$ M. J. Cabrita $\cdot$ R. Garcia $(\bowtie)$

ICAAM-Instituto de Ciências Agrárias e Ambientais

Mediterrânicas, Universidade de Évora, Núcleo da Mitra, Ap. 94,

7002-554 Évora, Portugal

e-mail: raquelg@uevora.pt

R. Garcia

e-mail: rmartagarcia@yahoo.com
}

The results obtained in this study seem to show that there is a correlation between the polymer structure (including its physical characteristics) and their binding properties.

Keywords Molecularly imprinted polymer (MIP) ·

Dimethoate $\cdot$ Deltamethrin $\cdot$ Scanning electron microscopy (SEM) $\cdot$ Rebinding studies

\section{Introduction}

Nowadays, molecular imprinting has emerged as a very relevant methodology to produce highly polymeric porous material which can be considered tailor-made synthetic materials possessing selective adsorption properties for specific target molecules, or a class of structurally related compounds [1-3]. Thus, the molecular imprinting technique has received considerable attention in the fields of polymer science and chemical analysis due to its predetermined selectivity for target molecules, high affinity achievement and robustness of recognition [3]. These polymeric materials are prepared by copolymerization of functional monomers and cross-linkers in the presence of an imprinting molecule that is used as a template leading to the formation of an artificially generated three-dimensional polymer network which has recognition sites complementary in size, shape and spatial orientation of the peripheral functionality of the template molecule [4]. These recognition sites mimic the binding sites of biological receptors such as antibody-antigen conjugates allowing one to circumvent some of the disadvantages like the stability, related to natural receptors. Additionally, these polymeric materials exhibit unique characteristics which could be considered beneficial in analytical applications, such as the ability to detect and quantify low analyte concentrations. To perform molecular imprinting, the non-covalent approach (or self-assembly approach) is one of the most frequently used polymerization 
techniques, allowing the preparation of molecularly imprinted polymers (MIPs) due to the simplicity of the procedure and the wide variety of monomers that can interact with almost any kind of commercially available template. However, this approach suffers from some drawbacks mainly related to equilibrium processes involved in template-monomer interactions, which must be displaced towards the formation of the template monomer complex by means of the use of a large amount of monomer leading to the formation of a heterogeneous binding site distribution $[4,5]$. The resulting polymer must be grounded and sieved in order to achieve particles with an appropriate diameter for this application, followed by a subsequent washing procedure which must be able to clean the MIP cavities and minimizing the template bleeding. MIPs have been developed for a variety of applications including chromatography $[6,7]$, catalysis $[8,9]$, solid-phase extraction $[5,10-13]$, sensor technology [14-16] and drug delivery and discovery processes $[17,18]$.

The increasing production and application of pesticides for agricultural purposes involves serious risk to the environment and human health due to either exposure or through residues in food and drinking water [19]. In order to ensure food safety, the development of selective, simple, rapid, cost-effective and reliable analytical methodologies able to detect with higher accuracy the presence of pesticides in trace levels, is crucial.

Recently, the use of the "Quick, Easy, Cheap, Effective, Rugged and Safe" (QuEChERS) sample preparation method [20] has been applied for large-scale pesticide residue analysis in several food matrices [21]. More recently, much attention has been devoted to the use of molecular imprinting for the development of new adsorbents for solid phase extraction (SPE) $[5,10,11,13]$ since the coupling of MIPs with SPE combines the advantages of both enhancement of template molecule recognition and traditional separation methods. Therefore, molecularly imprinted solid phase extraction (MISPE) combines the high specificity, selectivity and sensitivity related to molecular recognition mechanisms with the high resolving capacity of separation methods. Imprinting technology connected with SPE has been used across a wide range of application areas, namely food matrices [5], biological samples [22] and environmental analysis [23].

The present work concerns the development of molecularly imprinted polymers using dimethoate $(\mathrm{dmt})$ and deltamethrin $(\mathrm{dm})$ as template molecules (Fig. 1) in order to evaluate their usefulness for the detection and quantification of these pesticides in food matrices. In the present study, pesticides belonging to the organophosphorus and pyrethroid classes have been studied. Pyrethroid pesticides are commonly used as replacements for the traditional organophosphorus pesticides used in agricultural practices.

To achieve this objective, the present paper reports the synthesis of several dimethoate and deltamethrin MIPs and their corresponding NIPs, which have been prepared using metacrylic acid (MAA) as the functional monomer and EGDMA and TEGDMA as cross-linkers, aiming to assess the specificity of the interactions. These porous materials were characterized using spectroscopic techniques (FTIR, NMR), termogravimetric analysis (TGA) and morphological microscopic techniques (SEM). Further chromatographic evaluation of the recognition performance of the imprinting systems has been also performed by means of imprinting factor measurements and affinity binding properties. The results obtained in this study can be seen as the basis for further studies concerning the optimization of the synthetic procedure of dmt- and dm-MIP in order to achieve selective SPE adsorbents for the extraction of pesticides from several food matrices.

\section{Materials and methods}

\section{Chemicals}

Methacrylic acid (MAA) (functional monomer), ethylene glycol dimethacrylate (EGDMA) and tetraethylene glycol dimethacrylate (TEGDMA) (cross-linkers), 1, 1' azobisisobutyronitrile (AIBN), including the analytical standards deltamethrin $(\mathrm{dm})$ and dimethoate $(\mathrm{dmt})$ (templates) were purchased from Sigma-Aldrich (Bellefonte, PA, USA) and were used without further purification. Dichloromethane for synthesis and acetic acid (glacial, $100 \%$ anhydrous) and methanol for washing steps were purchased from VWR International S.A.S. (Fontenay-Sons-Bois, France) and all the chemicals were used without purification, except dichloromethane which was dried by distillation before use. HPLC grade acetonitrile and methanol and acetone were purchased from VWR International S.A.S. (Fontenay-Sons-Bois, France) and Merck (Darmstadt, Germany), respectively. The water used in all experiments was distilled and purified by a Milli-Q system (Millipore, Bedford, MA, USA).

\section{Instrumentation}

An Hamamatsu L9588-06 Spot Light Source UV-lamp was used for the photo-polymerisations.

Elemental analysis was measured with a Carlo Erba 1108 Elementar Analyzer. For the morphological studies, a scanning electron microscopy (SEM) on a Hitachi S-3700 N instrument was used, with an accelerating voltage set to $15 \mathrm{kV}$. Samples were mounted on aluminium stubs using carbon tape and were gold coated. Fourier transform infrared spectroscopy (FTIR) analysis measurements were performed on a PerkinElmer Spectrum Two infrared spectrophotometer. In the case of the physico-chemical studies, the thermogravimetric analysis (TGA) was determined on a PerkinElmer STA 6000 at a heating rate of $20{ }^{\circ} \mathrm{C} \mathrm{min}-1$ up to $600{ }^{\circ} \mathrm{C}$ under a 


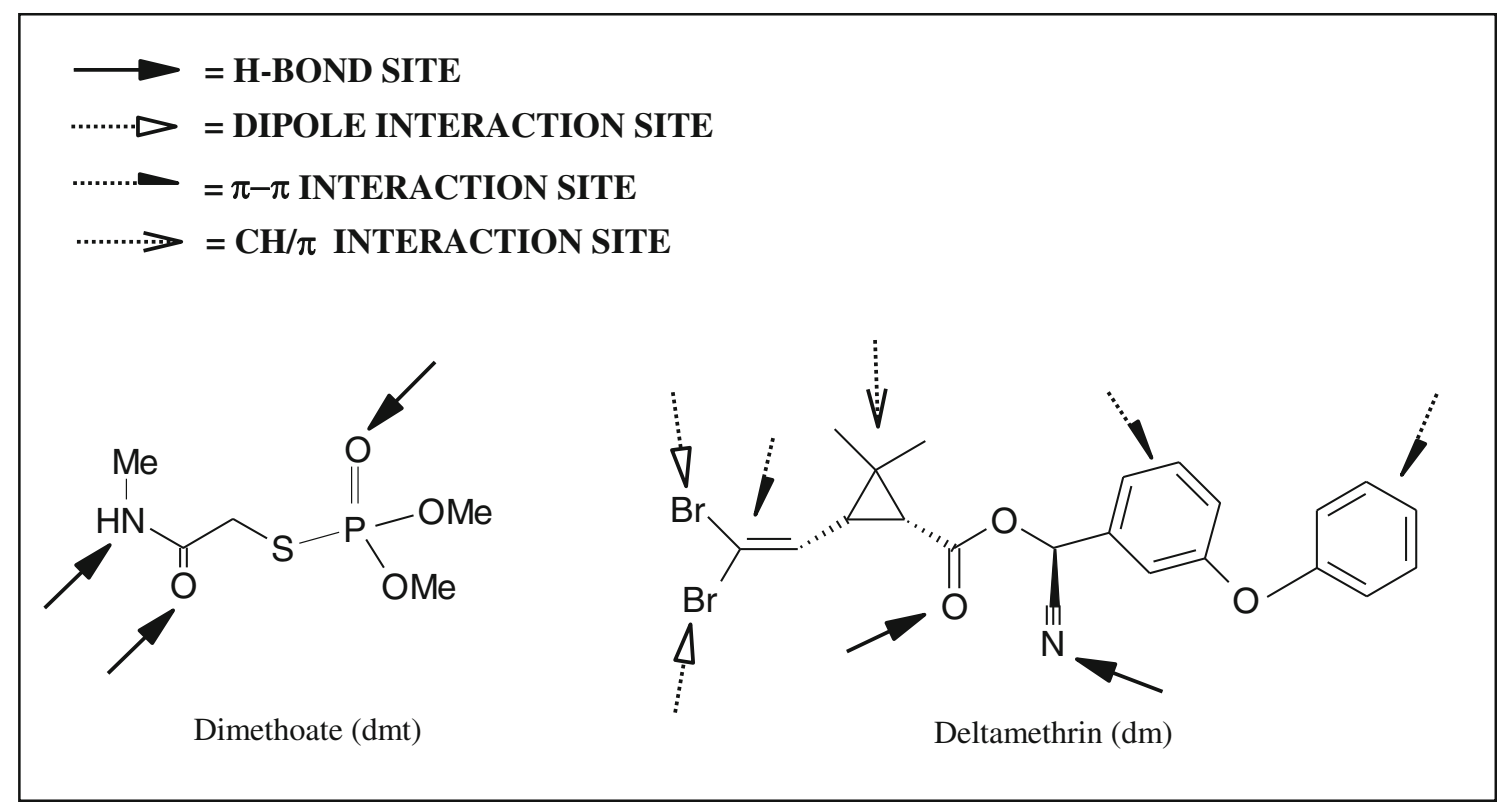

Fig. 1 Chemical structures containing assignments of possible interaction sites of the template molecules studied in this work

helium atmosphere. NMR solid state spectra were recorded on a Bruker Avance 500 spectrometer operating at $500 \mathrm{MHz}$ for phosphorus. The GC experiments were performed on an Agilent/HP 6890 instrument under the following conditions: chromatographic column, fused silica capillary column HP5, $5 \%$ phenyl methyl siloxane, $30 \mathrm{~m}, 0.25 \mathrm{~mm}$ I.D. and $0.25 \mu \mathrm{m}$ film thickness (Agilent GC column); chromatographic method, GC grade helium as carrier gas at a flow rate (constant flow) of $1.0 \mathrm{~mL} \mathrm{~min}{ }^{-1}$, column temperature program, $50{ }^{\circ} \mathrm{C}$ (held $5 \mathrm{~min}$ ) heated at $10^{\circ} \mathrm{C} \mathrm{min}^{-1}$ to $100^{\circ} \mathrm{C}$ (held for $10 \mathrm{~min}$ ) and then heated at $40{ }^{\circ} \mathrm{C} \min ^{-1}$ to $200{ }^{\circ} \mathrm{C}$ (held for $7.5 \mathrm{~min}$ ); injector temperature was set at $250^{\circ} \mathrm{C}, \mathrm{H}_{2}$ flow and air flow at $40 \mathrm{~mL} \mathrm{~min}^{-1}$ and $400 \mathrm{~mL} \mathrm{~min}^{-1}$, respectively. Detection was carried out by flame ionization.

Chromatographic evaluation of MIPs and NIPs, namely, imprinting factor determinations, was performed using an LC system LC30 (Dionex Corp., Sunnyvale, CA, USA) with a GP50-2 Gradient Pump and a LC30 column oven. Chromatographic experiments were carried out using a stainless-steel column $(50 \mathrm{~mm} \times 4.6 \mathrm{~mm})$ (Grace, Brandstraat, Belgium) packed with the synthesized MIPs and NIPs. Detection was carried out using a UV-vis detector (UVD $170 \mathrm{U}$ ) at $220 \mathrm{~nm}$. Output signals were monitored and integrated using Chromeleon 6.30 software.

Batch rebinding studies were performed using an HPLC UltiMate 3000 (Dionex Corp., Sunnyvale, CA, USA) system with LPG-3400SD gradient pump, TCC-3000SD column compartment and WPS-3000SL autosampler injector. Chromatographic experiments were carried out with a C18 Nucleosil reverse phase column $(250 \times 4.6 \mathrm{~mm}$ ID, $5 \mu \mathrm{m})$ (Grace, Brandstraat, Belgium). Detection was carried out using a photodiode array detector (DAD-3000RS) set in the range of 200-600 nm. Chromeleon 7.0 software was used for management, acquisition and treatment of data. On adsorption studies a VMS-C10 magnetic hotplate stirrer (VWR, Germany) and an Universal 32 centrifuge (Hettich Zentrifugen, Germany) were also used.

Synthesis of molecularly imprinted polymers

Molecularly imprinted polymers (MIPs) were synthesized using a molar ratio of template, functional monomer and cross-linker of 1:4:20 (Fig. 2). The functional monomer methacrylic acid (MAA, $1 \mathrm{mmol}$ ), the cross-linker, ethylene glycol dimethacrylate (EGDMA, $5 \mathrm{mmol}$ ) or tetraethylene glycol dimethacrylate (TEGDMA, $5 \mathrm{mmol}$ ) and either the dimethoate $(\mathrm{dmt}, 0.25 \mathrm{mmol})$ or deltamethrin template $(\mathrm{dm}$, $0.25 \mathrm{mmol}$ ) along with $1,1^{\prime}$-azobisisobutyronitrile (AIBN, $0.06 \mathrm{mmol}$ ) were dissolved in dry dichlorometane $(4 \mathrm{~mL})$ in a $10-\mathrm{mL}$ thick-walled glass tube. The mixture was degassed by flowing nitrogen through the flask for $10 \mathrm{~min}$ in an ice bath, and then stirred under irradiation from the UV lamp (at $240-400 \mathrm{~nm}$ ). After $24 \mathrm{~h}$, the polymer monolith obtained was ground and sieved in order to give particles ranging from $150 \mu \mathrm{m}$ to $300 \mu \mathrm{m}$. The particles were collected and washed extensively in a Soxhlet extractor with methanol/acetic acid solution $(9: 1(\mathrm{v} / \mathrm{v}))$ for $48 \mathrm{~h}$.

The non-imprinted polymers (NIPs) were synthesized using the same procedure, but in the absence of template.

Physical and morphological characterization

The morphological evaluation of the dimethoate- and deltamethrin-imprinted polymers was performed by scanning 
electron microscopy (SEM). The physico-chemical characterization was achieved using spectroscopic (FTIR, NMR) techniques, thermogravimetric analysis and microanalysis.

\section{Chromatographic evaluation}

\section{Imprinting capacity}

MIP1-MIP4 and NIP1-NIP2 were crushed and sieved to obtain particles of uniform size $(<45 \mu \mathrm{m})$, then the polymer powder (nearly 250 to $350 \mathrm{mg}$ ) was suspended in acetonitrile and the slurry obtained was packed manually into a stainless steel HPLC column $(50 \mathrm{~mm} \times 4.6 \mathrm{~mm}$ I.D.). The column was connected to the HPLC system equipped with a UV detector, and the template residue molecules were removed from the column by continuously washing with methanol-acetic acid $(8: 2(\mathrm{v} / \mathrm{v}))$ until a stable baseline was reached. One hour prior to the first injection, the column was equilibrated with the eluent and for $10 \mathrm{~min}$ between injections. The elution profile study for the various analytes using MIP1-MIP4 and NIP1-NIP2 as stationary phases in HPLC was performed using solvents of different polarities with different flow rates (as discussed in Section 3.2.1) in order to gain insight into the optimal elution conditions.

Imprinting factor assays of MIP1-MIP4 and NIP1-NIP2 were carried out at ambient temperature by injection of a volume of $10 \mu \mathrm{L}$ of different template solutions ( $\mathrm{dmt}$ and $\mathrm{dm}$ ) in acetonitrile $(2 \mathrm{mM})$ and $20 \mu \mathrm{l}$ of acetone as the non-retained component (termed the void volume marker) for the determination of the void fraction of the column. The retention times of each substance were recorded at $220 \mathrm{~nm}$ with an isocratic flow rate using different mobile phases. Retention factor $(\mathrm{K})$ was calculated for each analyte as $\mathrm{K}=\left(t-t_{0}\right) / t_{0}$, where $t$ is the retention time of the analyte and $t_{0}$ the retention time of acetone. Following this, imprinting factors (IF) for each analyte were calculated from the capacity factors of each analyte obtained on the MIP1-MIP4 and NIP1-NIP4 columns subjected to the same chromatographic conditions, using the equation IF $=\mathrm{K}_{\mathrm{MIP}} /$ $\kappa_{\text {NIP }}$, where $\kappa_{\text {MIP }}$ and $\kappa_{\text {NIP }}$ are the retention factors of the analyte on the MIPs and NIPs, respectively. The imprinting factor experiments were done in triplicate.

\section{Batch rebinding studies}

Binding properties of MIP1-MIP4 and NIP1-NIP2 were evaluated by batch rebinding studies for each polymer. Those assays were performed following the above procedure: $20 \mathrm{mg}$ of the imprinted (MIP1-MIP4) or non-imprinted (NIP1-NIP2) polymers were weighed into $10-\mathrm{mL}$ glass vials followed by the addition of $2 \mathrm{ml}$ of acetonitrile, and then aliquots of different solutions of the template under study were added (final template concentrations range 0.0024$\left.0.36 \mathrm{mg} \mathrm{mL}^{-1}\right)$. Next, the vials were stirred at room temperature with a magnetic hotplate stirrer at $450 \mathrm{rpm}$. After $24 \mathrm{~h}$, the vials were centrifuged and the supernatant was removed carefully and analyzed by HPLC in order to measure the amount of free template (unbound to the polymers). The binary mobile phase consisted of solvents $\mathrm{A}$ (water) and $\mathrm{B}$ (acetonitrile) as follows: $25-100 \% \mathrm{~B}$ from 0 to $7 \mathrm{~min}$, then $100 \%$ B from 7 to 14 min, followed by $100-25 \%$ B from 19 to $24 \mathrm{~min}$. The flow rate was fixed at $0.5 \mathrm{~mL} \mathrm{~min}^{-1}$ during the entire chromatographic process. The injection volume was $25 \mu \mathrm{L}$, DAD detection was done at $220 \mathrm{~nm}$ and the detection was set between 200 and $600 \mathrm{~nm}$ to monitor the UV-vis absorption. The quantification of the template was performed by measuring the peak area and by means of the correspondent calibration curve. For the determination of the calibration curve a number of HPLC standard solutions were prepared and analyzed by HPLC. These were obtained by dilution of a stock solution of the corresponding template in the range $1.961 \times 10^{-3}-0.4125 \mathrm{mg} \mathrm{mL}^{-1}(\mathrm{dmt})$ and $1 \times 10^{-4}-0.38 \mathrm{mgmL}^{-1}$ $(\mathrm{dm})$ and the LOD and LOQ values obtained allowed the accurate quantification of the pesticides in the study. All the experiments were conducted in duplicate, and the average value was taken. The amount of template bound to the MIP $\left(\mathrm{S}_{\mathrm{b}}\right)$ was calculated by subtracting the amounts of free template $\left(\mathrm{S}_{\mathrm{f}}\right)$ at equilibrium from the initial solution $\left(\mathrm{S}_{\mathrm{i}}\right)$.

\section{Results and discussion}

\section{Synthesis of imprinted polymers}

A series of molecularly imprinted polymers (MIP1-MIP4) were synthesized using the functional monomer methacrylic acid (MAA), the cross-linker, ethylene glycol dimethacrylate (EGDMA) or tetraethylene glycol dimethacrylate (EGDMA) and either the pesticide dimethoate or deltamethrin as templates, as illustrated schematically in Fig. 2. AIBN was used as the initiator and the photo-polymerization was achieved using a UV lamp during $24 \mathrm{~h}$. Since it was observed that reaction times lower than $24 \mathrm{~h}$ did not lead to complete polymerization, $24 \mathrm{~h}$ was selected as the optimized reaction time. For the synthesis of all the MIPs in this study, the standard ratio of template:monomer:cross-linker used was 1:4:20, according to the standard conditions described by Chapuis and co-workers [24]. Recent studies by Tom and co-workers [25] have shown that the presence of a slight excess of MAA in solution during the polymerization step may optimize the number of interactions between the template molecule and the MAA functional groups, which ultimately result in the formation of the binding sites within the imprinted material. The same study has shown that a typical template:cross-linker ratio of 1:40 was used for the preparation of non-covalent MIPs since this provides rigidity in the polymer network ensuring a cavity that is complementary in shape as well as functionality to the template [25]. 


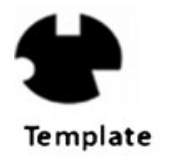

(dmt, dm)

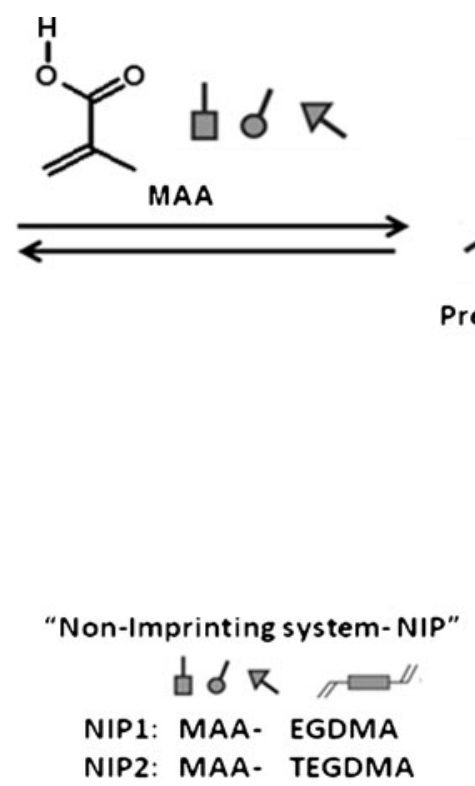

Fig. 2 Schematic representation of the MIP synthesis

The chemical structures of the templates under study (dmt and $\mathrm{dm}$ ) have influenced our synthetic strategy since dmt are flat molecules, whilst $\mathrm{dm}$ is a chiral molecule with three stereogenic centres (Fig. 1). In the case of dmt, which is a very small molecule with three key points for hydrogen bonding or dipolar interactions with monomers and the crosslinker, then the choice of monomer was limited to small molecules with high potential for hydrogen bond formation, thus MMA was selected as the monomer. The functional monomer plays a relevant role on the MIP molecular recognition performance since stronger interaction between template-monomer is crucial for the production of a highly stable complex and consequently higher recognition specificity of the MIPs [26]. According to several studies [26-28] MAA has been considered the optimal monomer when compared with other common functional monomers like 4vinylpyridine (4-VP) and acrylamide (AAm), since it leads to the formation of molecularly imprinted polymers with the highest binding capacities.

In the case of the cross-linker, both EGDMA and TEGDMA are adequate for the imprinting process providing the formation of cavities with suitable size to accommodate $\mathrm{dmt}$ and $\mathrm{dm}$ templates. For the imprinting process, dichloromethane was used as the porogen as it can bind to the dmt and $\mathrm{dm}$ templates via hydrogen bonding and dipole-dipole interactions and at the same time, since it is a small molecule, and it will not greatly disturb the resulting imprinting process, nor lead to excessively large cavities. The influence of the porogen on the strength of the non-covalent interactions and on the polymer morphology is well known, as it is responsible for creating the pores in the MIPs thus assuring reasonable "flow through" properties within the macroporous structure
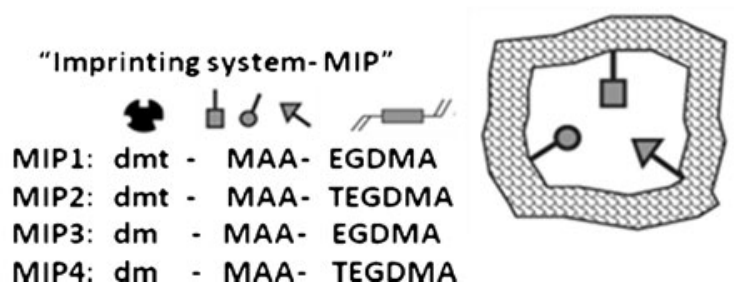
complex

$[2,29]$. Obviously, the choice of porogen is limited to the solubility of all the entities (M, template, initiator and $\mathrm{CrL}$ ) involved in the imprinting system.

Afterwards, the polymeric products were collected and washed by Soxhlet extraction using a methanol/acetic acid solution $(9: 1(\mathrm{v} / \mathrm{v}))$ for $48 \mathrm{~h}$. This extraction procedure is also appropriate to remove the remaining AIBN residues or unreacted monomer starting materials. This is considered the common method for washing the MIPs. Some of the nonwashed MIPs were retained for comparative analytical studies (see below). The polymers were all obtained as pale white solids.

Optimization of the MIP washing step and analysis of residual pesticide

Since bleeding phenomena constitute some of the main drawbacks associated with the imprinting systems [2], our first objective was to determine the efficiency of the template extraction process using microanalysis, the results of which were corroborated by other analytical techniques (FTIR, NMR). Microanalysis gave an indication of the carbon, hydrogen and nitrogen composition of the washed imprinted systems (MIP1-MIP4). Although, to study the efficiency of the template extraction process, this analysis was able to quantify more accurately the percentage of the template remaining in the imprinted cavities of the MIPs after the washing procedure. Unfortunately, it was impossible to remove all the template from the MIP, and depending on the MIP, the extraction system and the template used, the residual quantity ranged from $5 \%$ to $15 \%$. Such problems of removing the last traces of templates have also been reported by other authors 
Fig. 3 Comparison of the washing efficiency using a variety of solvent mixtures

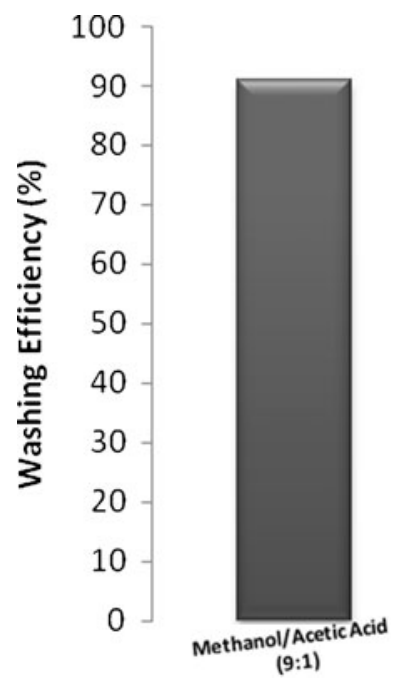

$[30,31]$. The presence of only residual amounts of template in the imprinted cavities demonstrated the importance of performing an efficient washing of the MIP, thus rendering the MIP cavities available for further analyte rebinding studies.

In order to optimize the MIP washing process, an extensive screening study of the Soxhlet Extraction process using several solvent mixtures was investigated (Fig. 3).

As depicted in Fig. 3, the most efficient washing solvent system was methanol/acetic acid $(9: 1(\mathrm{v} / \mathrm{v}))$ which had an efficiency of $91 \%$ for the extraction of the template from the imprinted polymer. This mixture performed very well due to its polar nature suiting the polar functional groups of the templates. Thus, methanol/acetic acid $(9: 1(\mathrm{v} / \mathrm{v}))$ was selected as the extraction solvent of choice.

Study of monomeric conversion during the polymerization step

The covalent bonds created between the functional monomer (M) and the cross-linker (CrL) are extremely important in order to maintain a stable polymer matrix for the imprinting process, but at the same time bestowing mechanical stability to the matrix [32]. The capacity and molecular recognition of the imprint binding sites owes much to the molar ratio $\mathrm{M}: \mathrm{CrL}$ which is responsible, together with the imprinting molecule, for the formation of a three dimensional cross-linked polymer
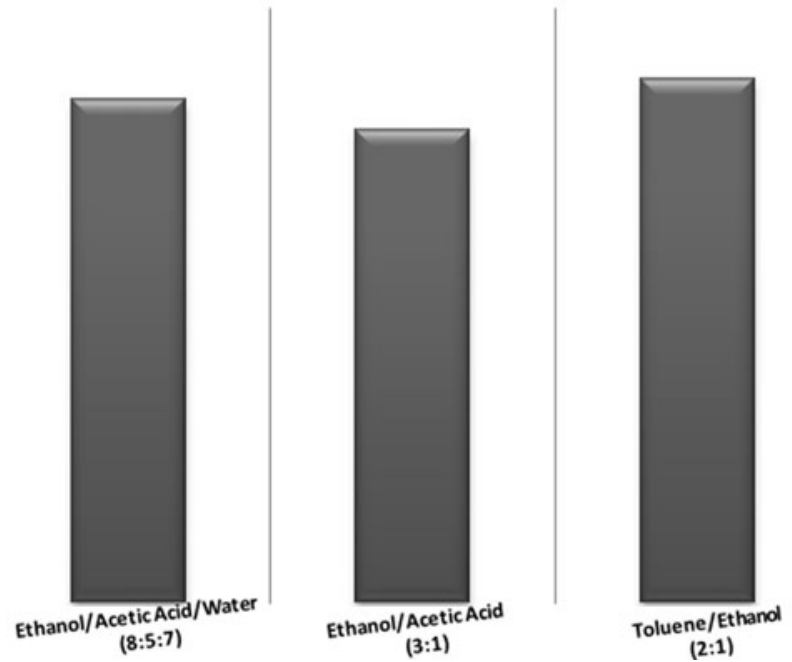

matrix with complementary binding features with regard to the guest molecule's shape, structure and functionality. In this study, NIPs and MIPs were synthesized having a molar ratio M:CrL of 1:5. In order to study the efficiency of the photopolymerization process the conversion of MAA during the imprinting process using gas chromatography (GC) was assessed, this study was carried out for the synthesis of NIP1. This study showed that during the photochemical synthesis, $97 \%$ of the MAA was converted to cross-linked polymer (NIP1). Thus, the synthetic method for the synthesis of all NIPs and MIPs was performed using a molar ratio $(\mathrm{M}: \mathrm{CrL})$ of $1: 5$.

\section{Morphological studies}

The performance of the MIPs is not exclusively dictated by the interaction of the template with the functional monomer pre- and post-polymerization at a molecular level, but also by their overall morphological features, which include the particle shape, size and the porous texture of the imprinting systems, which can affect the subsequent molecular recognition properties. Therefore the use of optical microscopy for the morphological characterization of the MIPs and NIPs [33] was crucial and for this purpose SEM, TEM and AFM analysis were used. The SEM technique is used quite frequently for this purpose as it is relatively simple to perform and constitutes a rapid form of analysis, and thus we wished to
Fig. 4 SEM micrographs of NIP1 (left-hand side) and NIP2 (right-hand side) at $10,000 \times$ resolution
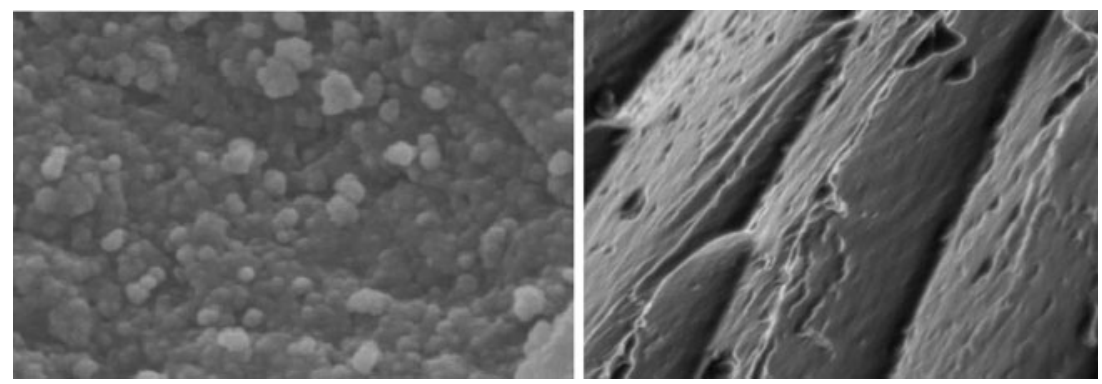
Fig. 5 SEM micrographs of MIP1 (left-hand side) and MIP2 (right-hand side) at 30,000× resolution
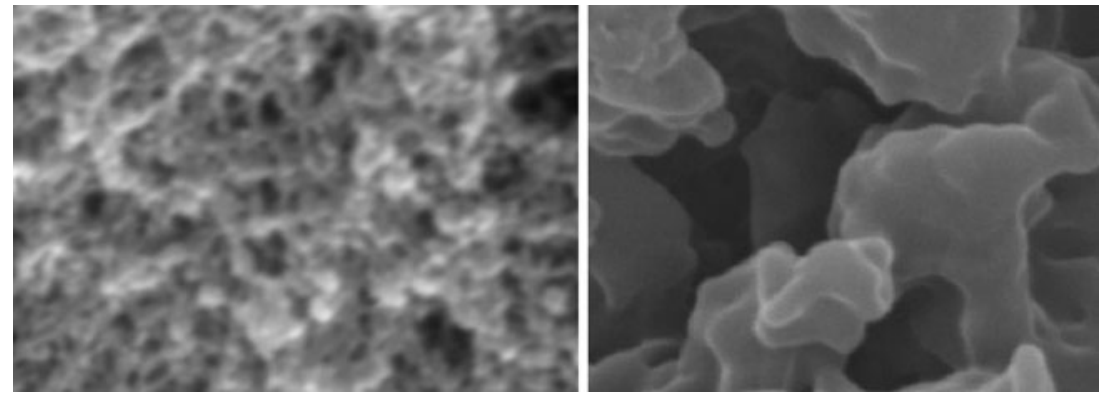

explore this technique for both morphological characterization of the MIPs and the NIPs and to try and gain an insight into the structure of the MIP cavities. Figures 4, 5 and 6 show the SEM micrographs obtained for several MIPs and NIPs synthesized in this work. Our studies using both TEM and AFM were without success.

Figure 4 presents the SEM images of NIP1 and NIP2. The two types of NIPs showed different morphological features reflecting the use of different cross-linkers on the synthesis of these porous materials. NIP1 seemed to have a more grainy surface texture than the NIP2 counterpart, which was much smoother. While NIP1 presents interconnected spherical and small globules that form a porous structure, NIP2 has a more compact arrangement. Overall, the results of the SEM analyses for the NIP systems confirm the importance of using a suitable cross-linking monomer, for controlling the polymeric matrix morphology [29].

SEM images of MIP1 and MIP2 (Fig. 5) show for the former the presence of cavities with sizes in the range 55$110 \mathrm{~nm}$, whilst for MIP2, cavity sizes between 190 and $420 \mathrm{~nm}$ were visible. Morphologically both MIPs have similar structures, but appreciable differences in their morphologies could be detected if compared with NIP structures showing the influence of the template on the overall polymer morphology.

SEM images of MIP3 and MIP4 (Fig. 6) showed that in the case of MIP3 there seem to be cavities in the range 120$200 \mathrm{~nm}$, whilst for MIP4, the cavities seem to be better defined, but bigger, ranging in size from 85-620 nm.

The results of the SEM analysis seems to indicate that MIPs containing TEGDMA as cross-linker have a more rigid and compact structure and the size of the cavities are greater than those obtained with EGDMA. SEM images also show that dimethoate imprinted polymers have a more uniform dispersion and quantity of imprinting cavities compared to deltamethrin imprinted polymers. Some of the morphological data obtained for MIPs and NIPs, in particular, the porous size measurement, has reflected a bigger average porous size for the MIP particles than for the NIP systems, suggesting some influence of the template on the particle growth during the polymerization.

\section{Chemical characterization}

In order to gain some insight into the interactions between the template and the MIP cavity inner wall, some FTIR studies for NIP1-NIP2 and MIP1-MIP4 were carried out. The infrared spectra of MIP1 (Fig. 7) and MIP2 (Fig. 8) before and after depletion of the template molecule, as well as that of the corresponding NIPs are depicted below.

The FTIR spectra showed very little difference between the NIP and undepleated MIP material: in either the case of the MIP1/NIP1 and MIP2/NIP2 systems (Figs. 7 and 8, respectively). The intense bands at about $1,700 \mathrm{~cm}^{-1}$ are undoubtedly attributed to $\mathrm{C}=\mathrm{O}$ stretching of the ester and acrylic acid whilst that at $c a .3,000 \mathrm{~cm}^{-1}$ can be attributed to methyl $\mathrm{C}-\mathrm{H}$ stretching. The fact that the bands due to the template could hardly be visualized is probably a consequence of the highly diluted nature of the template within the polymer structure. The adsorption peak around $1,500 \mathrm{~cm}^{-1}$ assigned to the stretching vibration of residual vinylic $\mathrm{C}=\mathrm{C}$ bonds, was present in both polymers (MIPs and NIPs). The main difference between the spectra was the presence of an absorption peak at $660 \mathrm{~cm}^{-1}(*)$ which belongs to the pesticide dimethoate
Fig. 6 SEM micrographs of MIP3 (left-hand side) and MIP4 (right-hand side) at $30,000 \times$ resolution
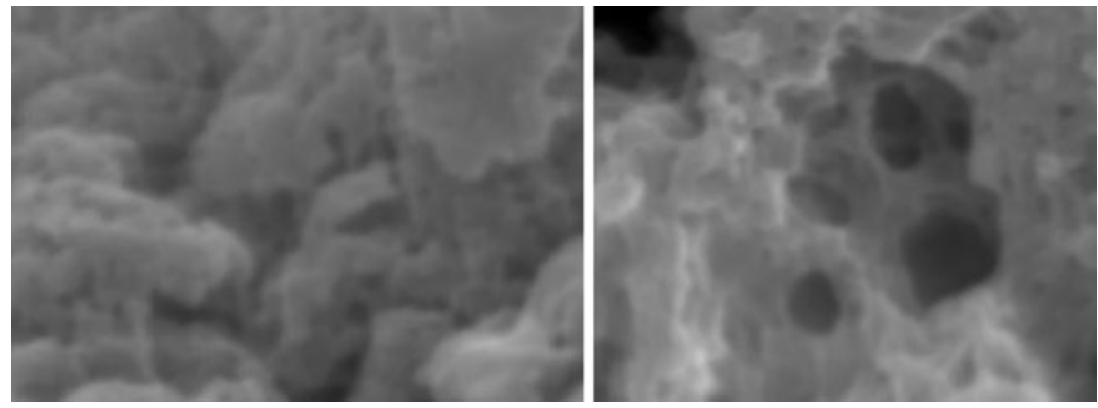


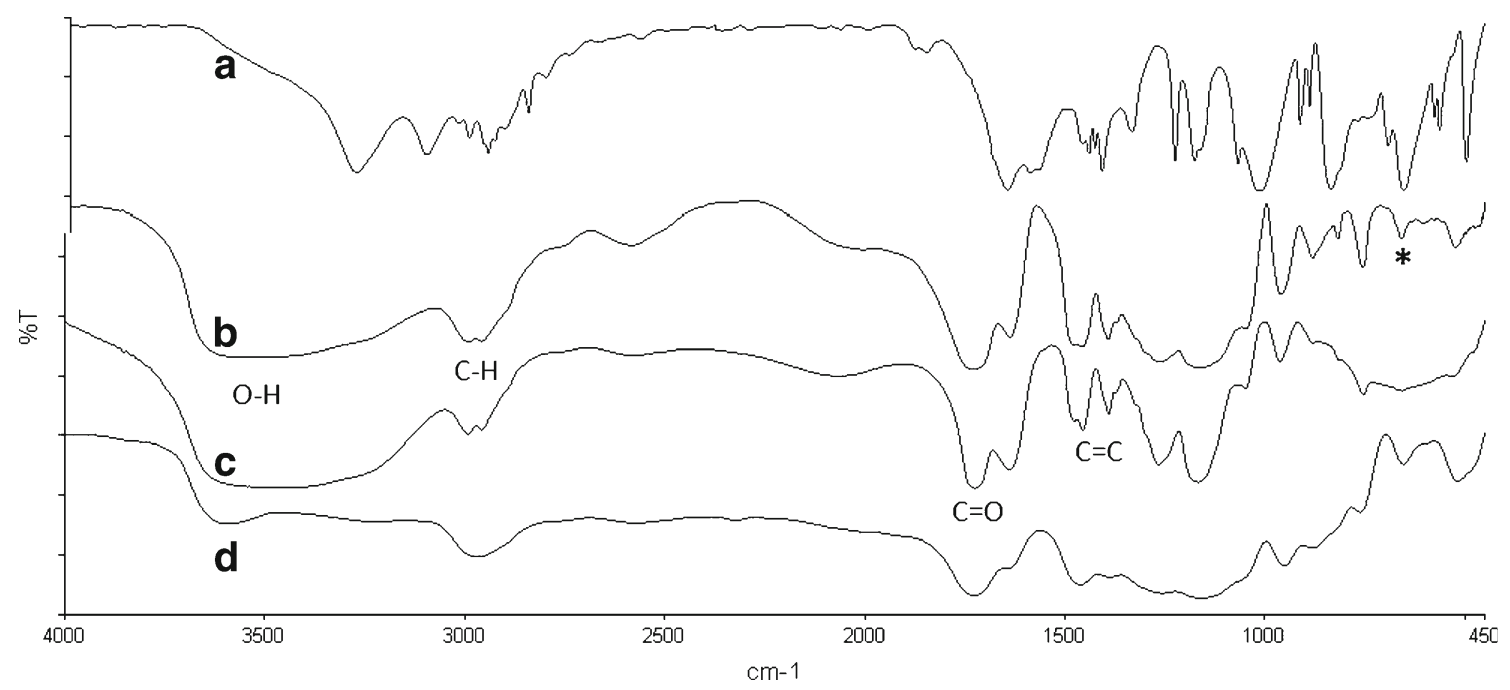

Fig. 7 FTIR spectra of (a) dmt template, (b) MIP1 before depletion of dmt, (c) MIP1 after depletion of dmt, and (d) NIP1

(Figs. 7 and $8 \mathrm{a}$ and b). The absence of that peak which is assigned to dmt in the depleted MIP spectra (Figs. 7c and 8c) seems to indicate the efficiency of the template removal from the imprinting systems in this study.

In an attempt to characterize fully the imprinted systems in this study we have performed a ${ }^{31} \mathrm{P}$ NMR study for the dimethoate imprinted MIPs (Fig. 9), aiming to obtain key structural information regarding the interaction of the template with the MIP cavity inner wall.

Preliminary studies indicate that the template was present in the cavities of the unwashed MIP, and that there was no alteration in the chemical shift for the ${ }^{31} \mathrm{P}$ nucleus remaining at $98 \mathrm{ppm}$. This fact was indicative of weak interactions between the phosphorous atom and the carboxylic acid side arms of the MIP cavities, and perhaps seemed to give some indications about the relative orientation of the template within the cavity, with perhaps the phosphorous containing part of the template being positioned distal to the cavity wall. The NMR data obtained shows clearly that most of the dmt had been removed from the MIP after washing. However, as this technique is less sensitive than microanalysis, the usefulness of this technique to determine the efficiency of removal of the pesticide during the washing step is, therefore, moderate. In order to evaluate the thermostability of the MIPs and understand some of its textural properties and mechanical properties, thermogravimetric analysis (TGA) studies were performed for all the MIPs and NIPs studied.

For the NIP systems, the decomposition of NIP1 started at $320{ }^{\circ} \mathrm{C}$ with total decomposition at $440{ }^{\circ} \mathrm{C}$ whilst for NIP2 it starts at a temperature of $235^{\circ} \mathrm{C}$ and loses total weight at $415^{\circ} \mathrm{C}$. Thus, the decomposition of NIP1 occurs at a higher temperature than NIP2 (TEGDMA-co-MAA), showing some evidence of its more stable and robust composition and structure. In the case of

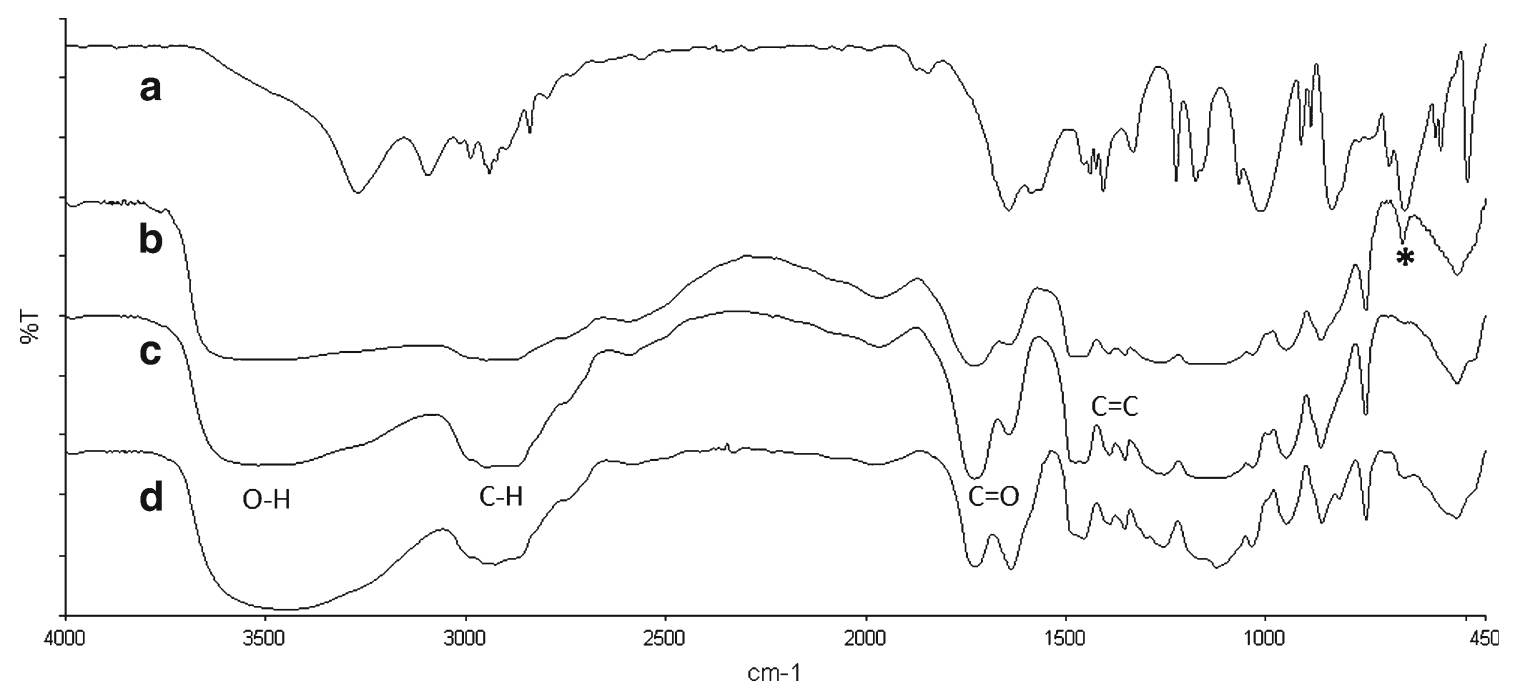

Fig. 8 FTIR spectra of (a) dmt template, (b) MIP2 before depletion of dmt, (c) MIP2 after depletion of dmt, and (d) NIP2 


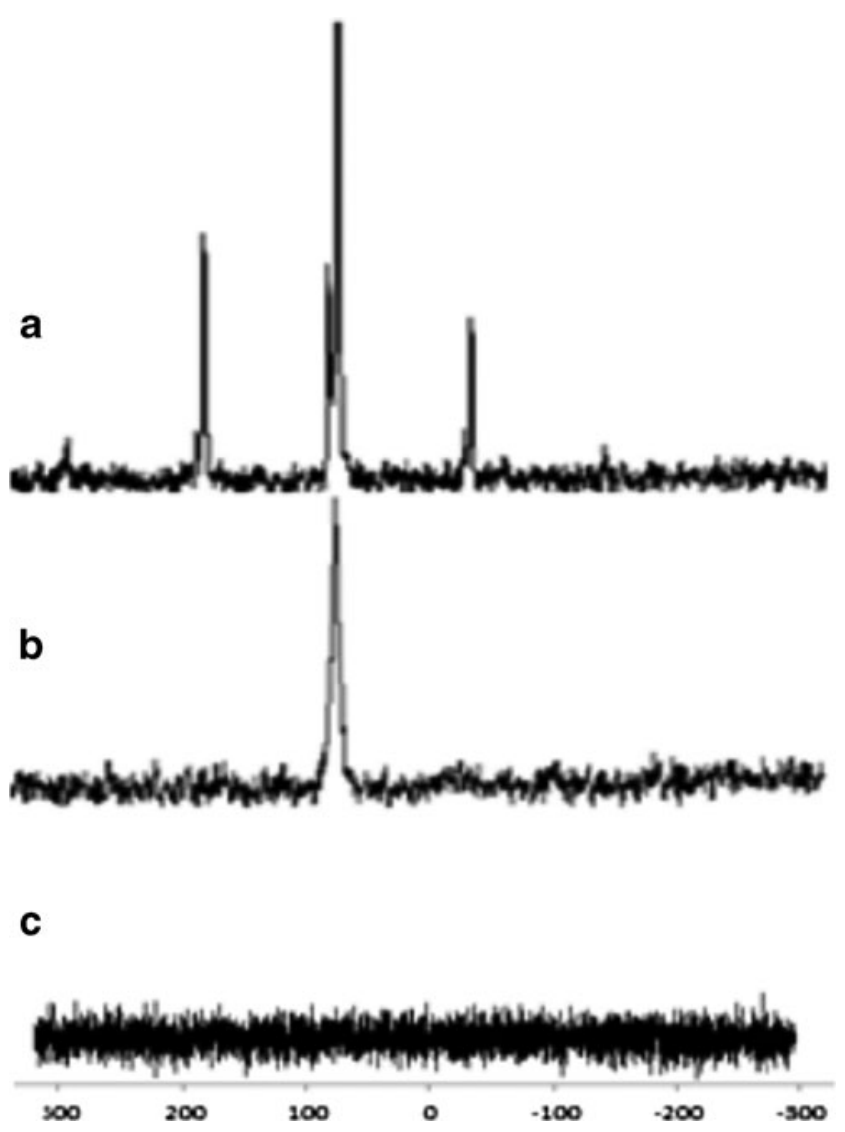

Fig. $9{ }^{31} \mathrm{P}$ NMR solid state spectra (500 MHz) of (a) dmt; (b) MIP1 before depletion of template; (c) MIP1 after depletion of template

MIP1 and MIP3 similar observations as regards the initial and final decomposition temperatures have been observed for NIP1. Equally, MIP2 and MIP4 have shown the same range of values of decomposition temperature than the respective NIP2. These results seem to indicate that, after depletion of the template from the polymeric material, the MIP shows the same thermogravimetric behavior as the NIP, hence the presence of $\mathrm{dmt}$ and $\mathrm{dm}$ in the templating process seems not to affect the structural or mechanical properties of the final MIPs.

Chromatographic evaluation

\section{Imprinting capacity}

Imprinting factors are a valuable tool for the evaluation of the usefulness of molecularly imprinted polymers as adsorbents for solid phase extraction (SPE). Because of the heterogeneous nature of MIP binding sites that have been prepared by the self-assembly approach, it is mandatory to apply reliable methodologies that could study the binding behavior of those imprinted materials. In particular, this evaluation comprises an experimental procedure in which MIPs are used in the high performance liquid chromatography (HPLC) stationary phase. The non-imprinted materials were also studied to

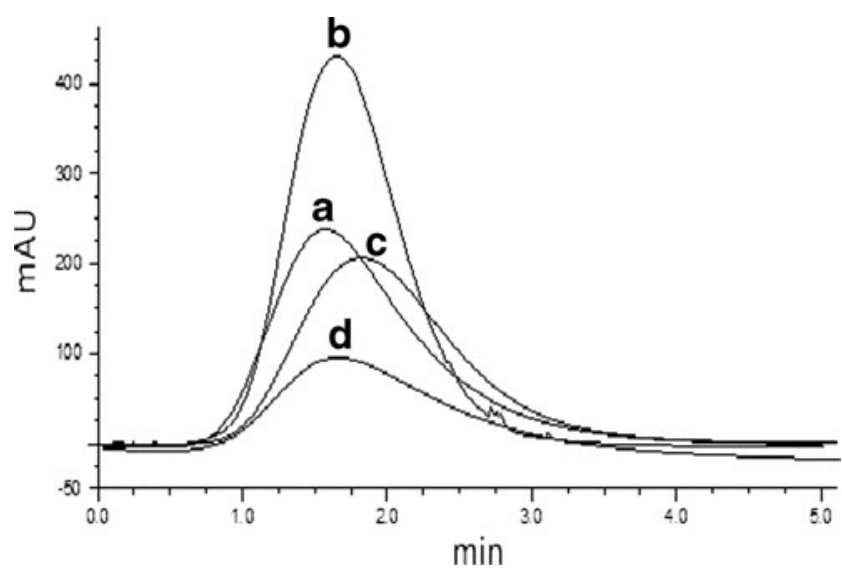

Fig. 10 Elution profile of dmt on MIP1 column using different mobile phases: (a) pure acetonitrile, (b) acetonitrile-water $(8: 2(\mathrm{v} / \mathrm{v}))$, (c) pure methanol and (d) methanol-water $(8: 2(\mathrm{v} / \mathrm{v}))$, flow rate was $0.5 \mathrm{~mL} \mathrm{~min}^{-1}$, $\lambda: 220 \mathrm{~nm}$

assess the specificity of the interactions. Thus, in this study, MIP1-MIP4 and NIP1-NIP2 were packed into a HPLC column in order to evaluate their specific absorption capability and recognition mechanism. The quantity of MIP used in the packaging step, the flow rate, the composition of the mobile phase as well as the injected pesticide volume have been optimized to provide an adequate elution profile, as shown in Fig. 10 for MIP1. Some differences in peak shapes, retention times and tailing (Fig. 10a-d) could be observed while the optimal elution was achieved using acetonitrile-water (8:2 $(\mathrm{v} / \mathrm{v}))$ as mobile phase (Fig. 10b), which increases the signal to noise ratio and the column efficiency.

As depicted in Fig. 10, peak tailing and broadening of the peak were observed when pure methanol and a mixture of methanol-water were used as mobile phase (Fig. 10c and d). However, the profile of the imprinted column peak is broader than that obtained with the conventional C18 column, an effect that has been also observed by other authors [34].

Table 1 Imprinting factors for MIP1-MIP4 stationary phases ${ }^{\mathrm{a}}$

\begin{tabular}{|c|c|c|c|c|}
\hline MIP & $\begin{array}{l}\text { Chromatographic conditions (eluent; flow } \\
\text { rate) }\end{array}$ & $\mathrm{K}_{\mathrm{NIP}}$ & $\mathrm{K}_{\mathrm{MIP}}$ & IF \\
\hline \multirow[t]{3}{*}{ MIP1 } & $\mathrm{MeOH} ; 1 \mathrm{~mL} \min ^{-1}$ & 0.04 & 0.13 & \\
\hline & $\mathrm{MeOH} / \mathrm{H}_{2} \mathrm{O}(90: 10(\mathrm{v} / \mathrm{v})) ; 0.5 \mathrm{~mL} \mathrm{~min}$ & 0.10 & 0.18 & \\
\hline & $\mathrm{CH}_{3} \mathrm{CN} ; 0.3 \mathrm{~mL} \mathrm{~min}^{-1}$ & 0.22 & 0.17 & 0.7 \\
\hline \multirow[t]{4}{*}{ MIP3 } & 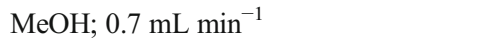 & 1.15 & 0.80 & 0.7 \\
\hline & $\mathrm{MeOH} / \mathrm{H}_{2} \mathrm{O}(90: 10(\mathrm{v} / \mathrm{v})) ; 1.0 \mathrm{~mL} \mathrm{~min}-1$ & 0.40 & 1.93 & 4.8 \\
\hline & $\mathrm{CH}_{3} \mathrm{CN} ; 0.7 \mathrm{~mL} \min ^{-1}$ & 0.03 & 0.05 & 1.6 \\
\hline & $\mathrm{CH}_{3} \mathrm{CN} / \mathrm{H}_{2} \mathrm{O}(90: 10(\mathrm{v} / \mathrm{v})) ; 0.5 \mathrm{~mL} \mathrm{\textrm {min } ^ { - 1 }}$ & 0.14 & 0.09 & 0.6 \\
\hline \multirow[t]{3}{*}{ MIP4 } & $\mathrm{CH}_{3} \mathrm{CN} ; 0.5 \mathrm{~mL} \min ^{-1}$ & 0.09 & 0.36 & 4.0 \\
\hline & $\mathrm{CH}_{3} \mathrm{CN} ; 0.7 \mathrm{~mL} \mathrm{~min}^{-1}$ & 0.11 & 0.06 & 0. \\
\hline & $\mathrm{CH}_{3} \mathrm{CN} / \mathrm{H}_{2} \mathrm{O}(90: 10(\mathrm{v} / \mathrm{v})) ; 0.5 \mathrm{~mL} \mathrm{\textrm {min } ^ { - 1 }}$ & 0.21 & 0.09 & \\
\hline
\end{tabular}

${ }^{\mathrm{a}}$ HPLC conditions: HPLC was an LC30 Dionex; MIP column was $50 \mathrm{~mm} \times 4.6 \mathrm{~mm}$ I.D.; injection volume was $10 \mu \mathrm{L} ; \lambda: 220 \mathrm{~nm}$ 


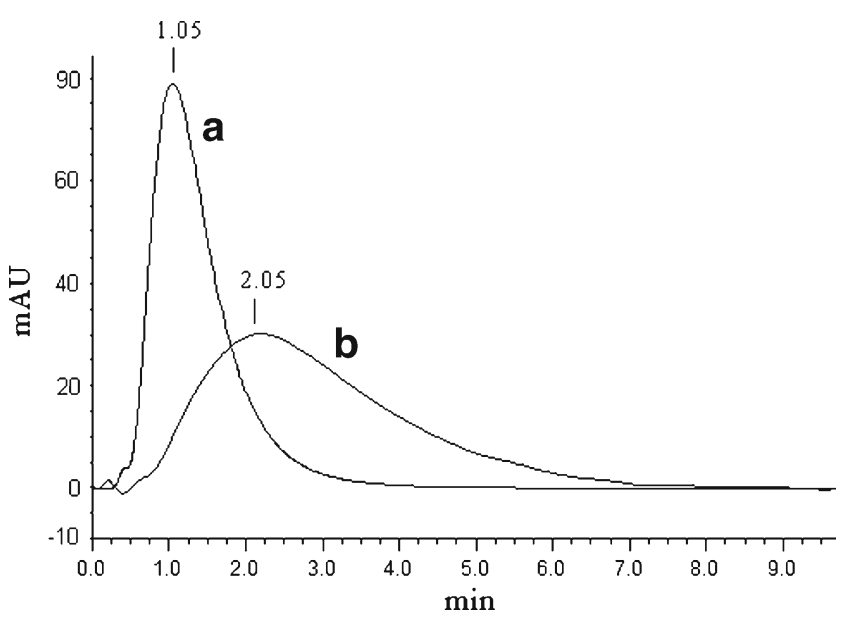

Fig. 11 Chromatographic profile of $\mathrm{dm}$ at (a) non-imprinted polymer (NIP1) and (b) imprinted polymer (MIP3); mobile phase was

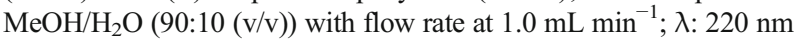

In order to measure the retention factors $(\mathrm{K})$ and imprinting factors (IF) for MIP1-MIP4, a screening study comprising the use of several mobile phases and different flow rates was performed for each MIP under study. The capacity factors were calculated from the adjusted retention times in order to avoid differences in retention time due to differences in packing efficiencies. Thus, the IF value reflects the imprinting effectiveness for the MIP column compared to its corresponding NIP. The results obtained in this study are summarized in Table 1.

Table 1 shows that the retention factors of the templates ( $\mathrm{dmt}$ and $\mathrm{dm}$ ) were longer with the MIP than the NIP column, which is the expected result since the NIP shows no recognition capacity for the template. For all the tested MIPs, MIP3 is the polymeric material that shows the best IF (4.83) for the following chromatographic conditions: eluent $\mathrm{MeOH} / \mathrm{H}_{2} \mathrm{O}$ $(90: 10(\mathrm{v} / \mathrm{v}))$, flow rate $1.0 \mathrm{~mL} \mathrm{~min}^{-1}$. Recently, $\mathrm{Xu}$ and coworkers [35] have introduced novel imprinting systems for the selective separation of deltamethrin using a $\beta$-cyclodextrin derivative as a functional monomer and toluene 2,4- diisocyanate as the cross-linker and the resulting studies were able to determine the molecular recognition of those systems, namely the imprint factors, which were in the range 1.992.44. Despite the use of different functional monomers and cross-linkers, the IF data obtained for MIP3 with deltamethrin as template is higher than the IF values of those imprinting systems. However, regarding imprinting systems for dimethoate recognition, the values obtained for MIP1 are relatively lower than the data described in other studies [36, 37]. Moreover, the porous intrinsic nature of the MIPs, together with several other factors, like shape recognition, hydrogen bond formation and hydrophophic interactions contribute strongly to the retention of the analyte, which is reflected in the varieties of IF values listed in Table 1 . In the case of the NIPs, their adsorption capacity was relatively weak compared to the corresponding MIPs which results from the intrinsic nature of the molecularly imprinted polymer as well as its molecular intrinsic affinity for the corresponding template. Figure 11 represents the elution profile of $\mathrm{dm}$ on the nonimprinted polymer (NIP1) (Fig. 11a) and on the imprinted polymer (MIP3) (Fig. 11b) columns.

As depicted in Fig. 11, the dm peak profile on the NIP column (Fig. 11a) is similar to its profile on the classic HPLC column, which exhibits a narrow peak without tailing. However, on the MIP column (Fig. 11b) the elution profile of $\mathrm{dm}$ is more broadened and with peak tailing when compared to the NIP column. This chromatographic behavior is characteristic of MIP columns and reflects the non-specific template adsorption pattern of NIP columns, that demonstrates easy elution by the mobile phase. These findings are in agreement with the results of other authors [34].

\section{Batch rebinding studies/adsorption studies}

Binding assays represent a key method for characterizing and comparing MIPs and is often used to evaluate the recognition mechanism of the synthesized MIPs [38]. To perform this
Fig. 12 Binding isotherms of MIPs (MIP1, MIP2) and NIPs (NIP1, NIP2) for the dmt template. Experimental conditions: mass of polymer, $20 \mathrm{mg}$; adsorption time, $24 \mathrm{~h}$

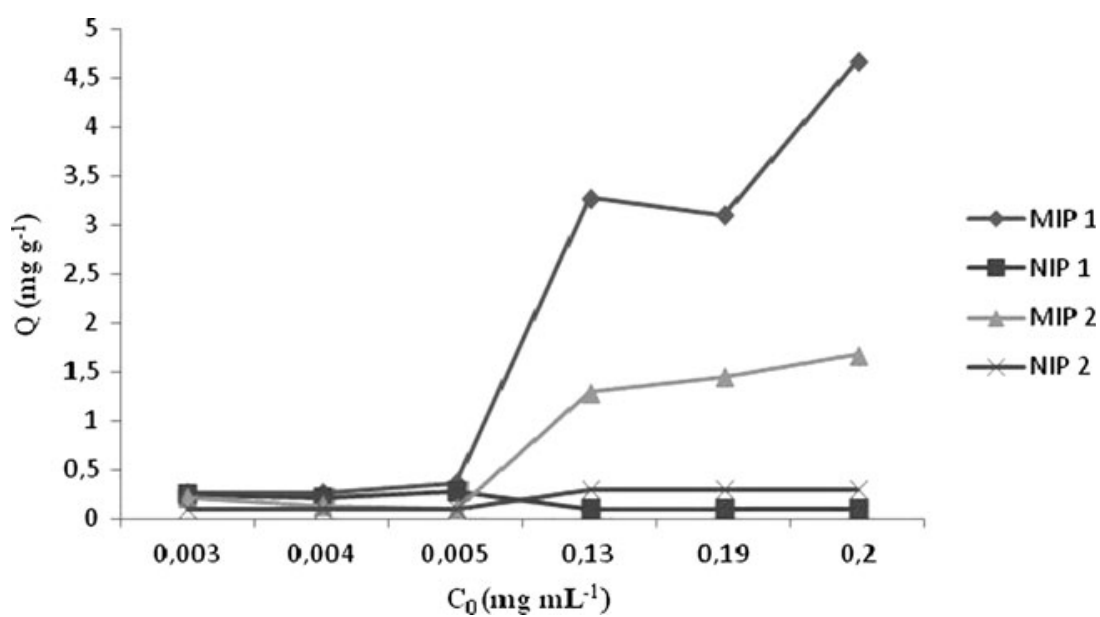


Fig. 13 Binding isotherms of MIPs (MIP3, MIP4) and NIPs (NIP1, NIP2) for dm template. Experimental conditions: mass of polymer, $20 \mathrm{mg}$; adsorption time, $24 \mathrm{~h}$

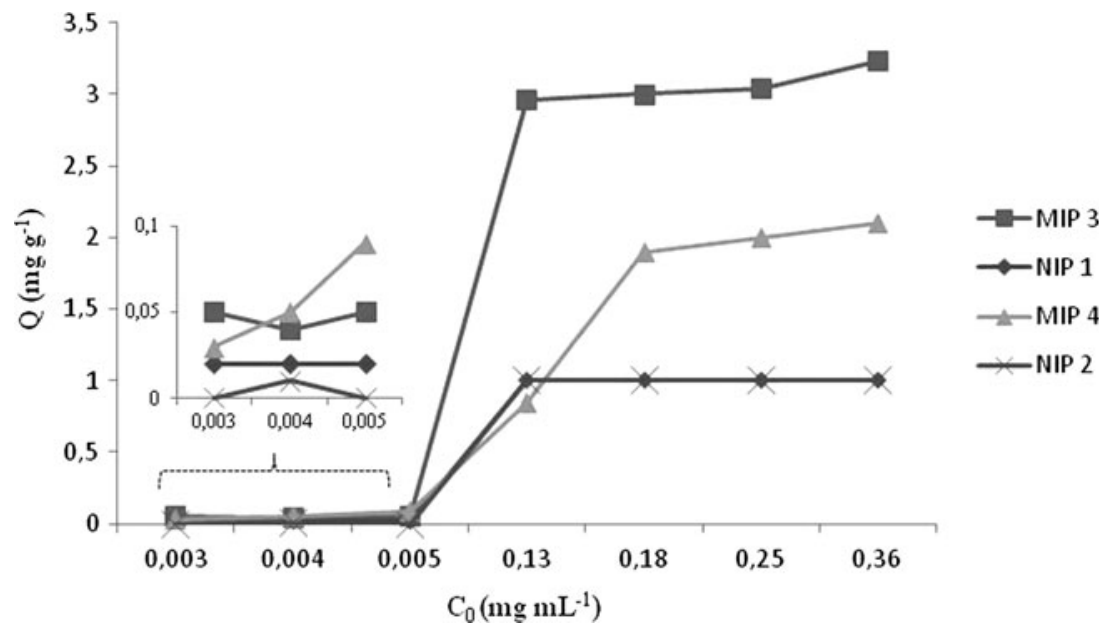

evaluation, adsorption isotherm experiments were carried out, which comprised the analysis of a heterogeneous mixture of the MIP with the corresponding template $(\mathrm{dmt}$ and $\mathrm{dm})$ at concentrations in the range of $3 \times 10^{-3}-0.36 \mathrm{mgmL}^{-1}$. In Figs. 12 and 13 the experimental results for the imprinted polymers MIP1-MIP2 and MIP3-MIP4, are depicted. The results of the rebinding studies for the corresponding NIPs (NIP1, NIP2) were also indicated for comparative purposes.

The adsorption isotherms represented in Figs. 12 and 13 measure the relationship between the equilibrium concentrations of bound and free template over a determined concentration range. As depicted in Figs. 12 and 13, the adsorbed template quantity is relatively lower when the template is present in minor concentrations because at those concentrations the template doesn't occupy all of the recognition sites of the imprinted material. The progressive increase in the concentrations of template solution leads to the occupancy of more and more recognition sites leading to a continuous increase of the polymer's binding capacity and at higher equilibrium concentrations than $0.13 \mathrm{mg} \mathrm{mL}^{-1}$ the binding capacity became stable for almost all the MIPs under study, with the exception of MIP1 that showed an increase in the binding capacity $\left(\mathrm{Q}_{\max }=4.5\right)$. Several authors have claimed (as well as stated by us above) that the molecular recognition properties of the imprinted polymers are intimately related to their morphology, or specifically in terms of the particle shape and size as well as the porous texture of the material [33]. Thus, MIP1 shows the highest binding capacity for the dmt template, which is correlated with their morphological structure obtained by SEM characterization. In particular, the SEM micrograph of MIP1 shows the presence of a microporous structure allowing the template to approach the binding sites (or cavities) located in the inner part of the polymer particles making their occupancy of the imprinted cavity easier. The relatively higher specific surface exhibited by MIP1 is another feature that could also explain the easier approach of the adsorbed template molecule. For the other MIPs (MIP2,
MIP3 and MIP4), the polymer particles are aggregated into more irregular structures and most of the binding sites are located in the interior of the imprinted material, thus complicating the entrance of the templates into the binding sites leading to a decrease in the binding capacity for the target analytes. The values of the binding capacities obtained for the different MIPs are in agreement with literature values [26]. Additionally, as depicted in Figs. 12 and 13, MIP1 and MIP3 having EGDMA as the cross linker, show higher binding capacity than their counterparts MIP2 and MIP4 which were prepared using TEGDMA as cross-linker. As expected, for the NIPs studied in this work there are no selective recognition sites and consequently the binding capacities were the lowest.

In order to evaluate the nature of the binding sites and cognizant of the fact that under non-covalent imprinting conditions, the resulting MIPs contain a heterogeneous mixture of cavities of varying affinity for the guest molecules, we have estimated the sorption capacity and equilibrium isotherm for the MIPs in this study, using Freundlich's isotherm (Eq. 1) as it is an empirical power function for non-ideal sorption on heterogeneous surfaces [4, 39-41].

$B=a C^{m}$
Table 2 Fitting parameters for the Freundlich adsorption isotherms of imprinted (MIP1-MIP4) and non-imprinted polymers (NIP1 and NIP2)

\begin{tabular}{lll}
\hline MIP & $m$ & $\mathrm{R}^{2}$ \\
\hline MIP1 & 0.5275 & 0.9914 \\
MIP2 & 0.6501 & 0.9809 \\
MIP3 & 1.0033 & 0.9665 \\
MIP4 & 0.8507 & 0.9819 \\
NIP1 & $0.2051^{\mathrm{a}}$ & $0.9250^{\mathrm{a}}$ \\
& $0.9287^{\mathrm{b}}$ & $0.9692^{\mathrm{b}}$ \\
NIP2 & $0.2774^{\mathrm{a}}$ & $0.9844^{\mathrm{a}}$ \\
& $1.1053^{\mathrm{b}}$ & $0.9464^{\mathrm{b}}$ \\
${ }^{\mathrm{a}}-\mathrm{dmt}{ }^{\mathrm{b}}{ }^{\mathrm{b}} \mathrm{dm}$ & & \\
\hline
\end{tabular}


where $B$ is the amount of template bound to the polymer, $C$ is the amount of free template in solution after equilibrium, $a$ is a Freundlich adsorption coefficient related with the binding affinity and $m$ is a Freundlich constant which represents the heterogeneity index and varies from 0 to 1 (values approaching 0 indicate increasingly heterogeneity and 1 is homogeneous). Freundlich's model is considered a generalization of the Langmuir model applied to a heterogeneous surface with an energy distribution corresponding to an exponential decrease [4]. The heterogenous nature of the polymer's binding sites diminishes the ability of the MIPs in almost every analytical application and, in particular, on chromatographic applications contributing to the broadening and asymmetry of the peaks.

Experimentally, the plot of $\log B$ vs. $\log C$ was applied to generate the intercept value of $a$ and the slope of $m$ by linear regression, assuming an exponential decaying sorption site energy distribution $[39,40]$. In Table 2 are summarized the experimental binding data relative to FI obtained for the MIPs studied in this work.

As indicated in Table 2, all the isotherms were in excellent agreement with an FI $\left(\mathrm{R}^{2}>0.95\right)$ over the concentration range described above. The heterogeneity indices $(m)$ for MIP3 and MIP4 were high, indicating that these imprinted polymers approached homogeneity in terms of binding site distribution. Moreover, this result was unexpected since all the polymers were prepared by a non-covalent approach leading typically to the production of heterogeneous binding sites on the crosslinker polymers due to the instability of the template-monomer complexes during the pre-polymerisation step. However, similar results have been recently described by Holland and co-workers [33]. In the case of MIP1 and MIP2 the $m$ values are lower than for MIP3 and MIP4, which seems to indicate the presence of heterogeneous binding sites on the cross-linker polymers with dimethoate recognition.

\section{Conclusions}

Dimethoate- and deltamethrin-tailor-made imprinted polymers have been prepared by a non-covalent photochemical imprinting approach using MAA as the functional monomer, EGDMA and TEGDMA as cross-linkers, dichlorometane as porogen and AIBN as initiator. The success of the polymerization process was established by means of physicomorphological techniques comprising the use of spectroscopic (FTIR, ${ }^{31} \mathrm{P}$ NMR), thermogravimetric (TGA) and microscopic (SEM) techniques. The molecular recognition of MIPs has been evaluated by imprinting capacity measurements and rebinding experiments, which show that our MIPs displayed adsorption capacity for the corresponding template. In order to evaluate the heterogeneous nature of the binding behavior of MIPs, an empirical Freundlich function for non-ideal sorption on heterogeneous surfaces has been applied and the heterogeneity indices for all the MIPs under study were determined. Despite the popularity of bulk polymerization for the preparation of molecularly imprinted polymers, the application of the resulting imprinted material for chromatographic evaluation is handicapped by the following principle aspect: the requirement for time consuming grinding and sieving of the material, in order to obtain particles within a homogeneous size distribution. Thus, we are currently exploring the possibility of preparing both $\mathrm{dmt}$ and $\mathrm{dm}$ molecularly imprinted polymers with alternative synthetic methods, which can predictably lead to the formation of well-defined polymers with low polydispersities, controlled composition and functionality, so that we can augment their impact and application in analytical chemistry.

Acknowledgments This work is funded by FEDER Funds through the Operational Programme for Competitiveness Factors-COMPETE and National Funds through FCT-Foundation for Science and Technology under the Strategic Projects PEst-C/Agr/UI0115/2011 and PEst-OE/QUI/ UI0619/2011 and Project PTDC/AGR-ALI/117544/2010. Prof. Peter Carrott and Ms. Louisa Marques are acknowledged for their assistance with the TGA study. Laboratory HERCULES at the University of Évora is acknowledged for the SEM analyses. Prof. João Rocha and the solid state NMR service at CICECO, University of Aveiro, Portugal are thanked for the solid state MAS- ${ }^{31} \mathrm{P}$ NMR study.

\section{References}

1. Bui BTSK (2010) Haupt Anal Bioanal Chem 398:2481-2492

2. Vasapollo G, Del Sole R, Mergola L, Lazzoi MR, Scardino A, Scorrano S, Mele G (2011) Int J Mol Sci 12:5908-5945

3. Garcia R, Cabrita MJ, Freitas AMC (2011) Am J Anal Chem 2:16-25

4. García-Calzón JA, Díaz-García ME (2007) Sens and Actuators B 123:1180-1194

5. Martín-Esteban A (2013) Trends Anal Chem 45:169-181

6. Ansell RJ, Kriz D, Mosbach K (1996) Curr Opin Biotechnol 7:89-94

7. Nunez O, Gallart-Ayala H, Martins CPB, Lucci P (2012) J Chromatogr A 1228:298-323

8. Alexander C, Davidson L, Hayes W (2003) Tetrahedron 59:20252057

9. Corma A (2004) Sci Eng 46:369-417

10. Kloskowski A, Pilarczyk M, Przyjazny A, Namiesnik J (2009) Anal Chem 39:43-58

11. Augusto F, Carasek E, Silva RGC, Rivellino SR, Batista AD, Martendal E (2010) J Chrom A 1217:2533-2542

12. Beltran A, Borrull F, Marcé RM (2010) TrAC Trends Anal Chem 29: $1363-1375$

13. Hu Y, Pan J, Zhang K, Lian H, Li G (2013) TrAC Trends Anal Chem 43:37-52

14. Moreno-Bondi MC, Navarro-Villoslada F, Benito-Pena E, Urraca JL (2008) Curr Anal Chem 4:316-340

15. Shimizu KD, Stephenson CJ (2010) Curr Opin Chem Biol 14:743750

16. Malitesta C, Mazzotta E, Picca R, Poma A, Chianella I, Piletsky S (2012) Anal Bioanal Chem 402:1827-1846

17. Cunliffe D, Kirby A, Alexander C (2005) Adv Drug Delivery Reviews 57:1836-1853

18. Rathbone DL (2005) Adv Drug Delivery Reviews 57:1854-1874 
19. Regulation (EC) No. 396/2005 of the European Parliament and of the council of 23 February 2005 on maximum residue levels of pesticides in or on food and feed of plant and animal origin and amending Council Directive 91/414/EEC, and subsequent updates, http://ec. europa.eu/food/plant/protection/pesticides/inex_en.htm. and Codex Alimentarius Committee on Pesticide Residues, Joint FAO/WHO Meeting on Pesticide Residues (last update 2009) http://www. codexalimentarius.net/mrls/pestdes/jsp/pest_q-e.jsp

20. Anastassiades M, Lehotay SJ, Stajnbaher D, Schenck FJ (2003) J AOAC Int 86:412-431

21. Wilkowska A, Biziuk M (2011) Food Chem 125:803-812

22. Okutucu B, Onal S (2011) Talanta 87:74-79

23. Pichon V, Chapuis-Hugon F (2008) Anal Chim Acta 622:48-61

24. Chapuis F, Pichon V, Lanza F, Sellergren B, Hennion MC (2004) J Chromatogr B 804:93-101

25. Tom LA, Schneck NA, Walter C (2012) J Chromatogr B 909:61-64

26. Xu S, Li J, Chen L (2011) Talanta 85:282-343

27. Matsui J, Kubo H, Takeuchi T (1998) Anal Sci 14:699-702

28. Siemann M, Andersson LI, Mosbach K (1998) J Agric Food Chem 44:141-145

29. Piletska EV, Guerreiro AR, Whitcombe MJ, Piletsky SA (2009) Macromolecules 42:4921-4928
30. Martin P, Jones GR, Stringer F, Wilson ID (2003) Analyst 128:345-181

31. Andersson LI, Paprica A, Arvidsson T (1997) Chromatographia 46: $57-66$

32. López MMC, Pérez MCC, García MSD, Vilarino JML, Rodríguez MVG, Losada L-FB (2012) Anal Chim Acta 721:68-78

33. Holland N, Frisby J, Owens E, Hughes H, Duggan P, McLoughlin P (2010) Polymer 51:1578-1584

34. Tang K, Chen S, Gu X, Wang H, Dai J, Tang J (2008) Anal Chim Acta $614: 112-118$

35. Xu ZF, Wen G, Kuang DZ, Zhang FX, Tang SP (2013) J Environ Sci Health Part B 48:336-343

36. Bakas I, Oujji NB, Moczko E, Istamboulie G, Piletsky S, Piletska E, Ait-Ichou I, Ait-Addi E, Noguer Rouillon TR (2013) J Chromatogr A 1274:13-18

37. Lv Y, Lin Z, Feng W, Zhou X, Tan T (2007) Biochem Eng J 36:221-229

38. Pap T, Horvai G (2004) J Chromatogr B 804:167-172

39. Corton E, García-Calzón JA, Díaz-García ME (2007) J Non-Cryst Solids 353:974-980

40. Umpleby RJ II, Baxter SC, Bode M, Berch JK Jr, Shah RN, Shimizu KD (2001) Anal Chim Acta 435:35-42

41. Rampey AM, Umpleby RJ, Rushton GT, Iseman JC, Shah RN, Shimizu KD (2004) Anal Chem 76:1123-1133 